\title{
Neutrophil gelatinase-associated lipocalin (NGAL) and matrix metalloproteinases as novel stress markers in children and young adults on chronic dialysis
}

\author{
Kinga Musial • Danuta Zwolińska
}

Received: 28 July 2010 /Revised: 1 September 2010 /Accepted: 8 September 2010/Published online: 19 September 2010

(C) The Author(s) 2010. This article is published with open access at Springerlink.com

\begin{abstract}
Phenomena related to chronic kidney disease, such as atherosclerosis, aggravate with the introduction of dialysis. Matrix metalloproteinases (MMP) and factors modifying their activity, such as their tissue inhibitors (TIMP) or neutrophil gelatinase-associated lipocalin (NGAL), take part in the matrix turnover and the endothelial damage characteristic for atherogenesis. However, there are no data on the associations between these parameters and other known pro-atherogenic factors, or on the impact of various dialysis modalities on them. The aim of our study was to assess the serum concentrations of NGAL, MMP-7, MMP-9, and TIMP-1, as well as their correlations with human heat shock proteins (Hsp90 $\alpha$, anti-Hsp60), endothelial dysfunction (sE-selectin), and inflammation (hSCRP) in pediatric patients chronically dialyzed. Twenty-two children on automated peritoneal dialysis (APD), 17 patients on hemodialysis (HD) and 24 controls were examined. The serum concentrations of NGAL, MMP-7, MMP-9, TIMP-1, Hsp90 $\alpha$, antiHsp60, and sE-selectin were assessed by enzyme-linked immunosorbent assay (ELISA). The median values of NGAL, MMP-7, MMP-9, TIMP-1, and MMP-9/NGAL ratio were significantly elevated in all dialyzed children vs. controls and were higher in HD than in APD. The values of MMP-9/ TIMP-1 and MMP-7/TIMP-1 ratios in the HD subjects were lower than those in the APD children. Hsp $90 \alpha$ and antiHsp60 predicted the values of NGAL, MMPs, and TIMP-1. Additionally, sE-selectin was a predictor of NGAL levels, whereas NGAL predicted the MMP and TIMP-1 concentrations. The increased concentrations of examined parame-
\end{abstract}

\footnotetext{
K. Musial • D. Zwolińska $(\square)$

Department of Pediatric Nephrology,

Wrocław Medical University,

M.Skłodowskiej-Curie 50/52,

50-369 Wrocław, Poland

e-mail: zwolin@nefped.am.wroc.pl
}

ters indicate the dysfunction of MMP/TIMP/NGAL system in the dialyzed children, more pronounced on hemodialysis. The discrepancies between dialysis modalities and correlations with heat shock proteins (HSPs) suggest that NGAL may be considered a novel stress protein, whereas MMP-7, MMP-9, and TIMP-1 may be regarded as indicators of stress response in the pediatric population on chronic dialysis.

Keywords Heat shock proteins $\cdot$ Hemodialysis $\cdot$ Peritoneal dialysis · Matrix destruction · Lipids · Endothelial damage

\section{Introduction}

Inflammation, lipid disturbances, endothelial damage, and autoimmunity play a pivotal role in atherogenesis (Blasi 2008; Wick et al. 2004). The acceleration of atherosclerosis is characteristic for the patients with chronic kidney disease, and the aggravation of that process concerns particularly those who are on dialysis (Andreoli et al. 2007).

Extracellular HSPs and their antibodies may also take part in atherogenesis (Rigano et al. 2007; Leng et al. 2010). The role for human HSPs in predicting the risk of acute coronary syndrome and accelerating atherosclerosis has been confirmed very recently (Dulin et al. 2010; Zhang et al. 2010). Moreover, generating anti-HSPs may be the result of immune response against both human and bacterial HSPs that have high-sequence homology (Pockley et al. 1999; Wu and Tanguay 2006). Such a cross-reactivity, excellently exemplified by Hsp60 and anti-Hsp60 (Cappello et al. 2009), may disturb the immune response by triggering reactions against own HSPs (Perschinka et al. 2003). Another contribution of HSPs to innate immunity is their ability to activate nuclear factor (NF)- $\mathrm{KB}$ - one of the potent stimulators of MMP and TIMP secretion. Both human anti- 
Hsp60 and Hsp90 $\propto$ exert such an activity (Schett et al. 1995; Madrigal-Matute et al. 2010).

Therefore, the HSP-anti-HSP system may have an impact on the disturbed turnover of extracellular matrix, which in turn is responsible for myocardial and vascular remodeling. Indeed, mounting evidence points toward noxious or protective effects of matrix metalloproteinases and their tissue inhibitors on the development of atherosclerosis (Johnson et al. 2005; Kuzuya et al. 2006).

MMPs represent a family of endopeptidases with the proteolytic activity, targeting the components of extracellular matrix. Their activity can be regulated by several factors, such as NF- $\mathrm{kB}$, cytokines or oxidative stress (Newby et al. 2009). Such a variety of control pathways may be responsible for their ambiguous effects. In detail, MMP-9 (gelatinase B) has been described in in vitro investigations as a protector of the plaque stability in its early stages and the promoter of the increased vulnerability through hemorrhage in the advanced lesions (Luttun et al. 2004; de Nooijer et al. 2006). MMP-9 is also one of the risk factors for myocardial infarction (Jefferis et al. 2010). TIMP-1 is a specific inhibitor of MMP9 , binding to it in a 1:1 proportion. The role of TIMP-1 in atherogenesis is not clear, but its concentration correlates positively with left ventricular mass and heart wall thickness (Hansson et al. 2009).

Neutrophil gelatinase-associated lipocalin (NGAL; lipocalin 2) is another specific MMP-9 binder, preventing it from degradation and, thus, maintaining its activity. However, lipocalin 2 occupies mainly an established position of a marker of severity of renal impairment (Bolignano et al. 2008; Mitsnefes et al. 2007; Nishida et al. 2010). Recent studies have also suggested its role in atherosclerosis and inflammation. NGAL expression has been reported in atherosclerotic plaques (Hemdahl et al. 2006), whereas the presence of MMP-9/NGAL complex has been suggested indicative of their erosion (Diamanti-Kandarakis et al. 2008). Moreover, NGAL activity is regulated by IL-1 $\beta$ and NF-KB (Bu et al. 2006; Cowland et al. 2006). The in vitro experiments have shown another side of the coin, because increased NGAL concentrations could protect against thermal stress (Roudkenar et al. 2009). However, the role of lipocalin 2 as a potential stress marker in humans has not been tested yet.

The elevated serum concentrations of MMP-7 (matrily$\sin$ ) were observed in patients with coronary artery disease (Nilsson et al. 2006). However, nothing is known about the role of MMP-7 in the subjects with chronic kidney disease, or in the patients on dialysis.

The data on the role of circulating MMPs/TIMPs in endstage renal disease are scarce and concern mainly adult patients on hemodialysis (Preston et al. 2002; Pawlak et al. 2005; Naganuma et al. 2008). Single studies have described the role of MMP-9, TIMP-1, and NGAL as markers of peritonitis in the patients on peritoneal dialysis (Fukudome et al. 2001; Leung et al. 2009). There are no data so far on these parameters in the pediatric patients on chronic dialysis or studies comparing these parameters in the subjects treated with different dialysis modalities.

Therefore, our aim was to assess the serum levels of NGAL, MMP-7, MMP-9, and TIMP-1 in children on peritoneal dialysis and hemodialysis, searching for the differences between these modalities. Secondly, we investigated the associations with human stress proteins (Hsp90 $\alpha$, antiHsp60), endothelial markers (sE-selectin), and the known markers of atherosclerosis, such as the lipid profile disturbances or inflammation (hsCRP), to assess the role of MMP/ TIMP/NGAL system in stress response, in the acceleration of atherosclerosis and in the differentiation between the various dialysis modalities.

\section{Materials and methods}

Sixty-three patients enrolled in the study were divided into three groups. Basic demographical data are put into the Table 1.

The first group consisted of 22 children on automated peritoneal dialysis (APD-Baxter, Home choice). The patients had ten exchanges during the night and, if necessary, one or two during the day. Peritoneal fluids used in our patients had glucose concentrations varying from $1.36 \%$ to $2.27 \%$. The causative factors in CKD were: chronic pyelonephritis (seven cases), chronic glomerulonephritis (six), polycystic kidney disease (two), neurogenic bladder (three), hemolytic uremic syndrome (two), and unknown (two).

The second group included 17 children and young adults hemodialyzed (HD) on polysulfone membranes. HD sessions (3.5-4 h) were performed three times a week, using bicarbonate dialysate, the blood flow ranged from 150 to $200 \mathrm{ml} / \mathrm{min}$, dialysate flow did not exceed $500 \mathrm{ml} / \mathrm{min}$. The membrane area was between 1.0 and $1.6 \mathrm{~m}^{2}$, the dialyzers were not reused. The water, purified by reosmosis, was regularly checked for contamination. All patients were on stable anticoagulation regimen using low molecular weight heparin. The causative factors in chronic renal failure were: chronic glomerulonephritis (six cases), chronic pyelonephritis (six), neurogenic bladder (two), polycystic kidney disease (one), and unknown (two).

To avoid the possible impact of previous glomerulopathy on the concentrations of examined parameters, the patients on dialysis were subdivided into those with chronic glomerulonephritis as an underlying cause of CKD (12 cases) and those with abnormalities within the urinary tract 
Table 1 The median values and interquartile ranges of basic demographical data and examined parameters in the patients on peritoneal dialysis (APD), on hemodialysis (HD) and in the control group

Mann-Whitney $U$ test:

${ }^{\mathrm{a}} p<0.01$ APD vs. control group

${ }^{\mathrm{b}} p<0.05$ APD vs. HD

${ }^{\mathrm{c}} p<0.01 \mathrm{HD}$ vs. control group

\begin{tabular}{llll}
\hline Parameter & \multicolumn{3}{l}{ Median values (interquartile ranges) of analyzed parameters } \\
\cline { 2 - 4 } & Control gr. $(n=24)$ & APD $(n=22)$ & HD $(n=17)$ \\
\hline Age (years) & $10.5(5.0-16.5)$ & $10.0(4.0-15.5)$ & $14.0(10.5-17.5)$ \\
Gender & 13 girls; 11 boys & 12 girls; 10 boys & 9 girls; 8 boys \\
Time of therapy (years) & - & $2.0(0.7-2.5)$ & $2.1(1.4-2.5)$ \\
Hsp90 $\alpha(\mathrm{ng} / \mathrm{ml})$ & $5.0(4.5-5.5)^{\mathrm{a}}$ & $28.5(20.0-36.0)$ & $25.0(20.0-25.0)^{\mathrm{c}}$ \\
Anti-Hsp60 $(\mathrm{ng} / \mathrm{ml})$ & $11.3(8.2-26.7)^{\mathrm{a}}$ & $49.3(14.85-71.85)^{\mathrm{b}}$ & $99.2(90.7-121.78)^{\mathrm{c}}$ \\
sE-selectin $(\mathrm{ng} / \mathrm{ml})$ & $26.1(22.2-28.0)^{\mathrm{a}}$ & $92.0(82.0-96.0)^{\mathrm{b}}$ & $104.0(94.0-108.0)^{\mathrm{c}}$ \\
hsCRP $(\mathrm{mg} / \mathrm{l})$ & $0.33(0.21-0.63)$ & $0.52(0.22-2.80)$ & $0.24(0.16-0.57)$ \\
Total CHOL $(\mathrm{mg} / \mathrm{dl})$ & $188.0(181.0-196.0)^{\mathrm{a}}$ & $221.0(212.0-275.0)$ & $220.0(180.0-250.0)$ \\
HDL-CHOL $(\mathrm{mg} / \mathrm{dl})$ & $62.0(59.0-67.0)^{\mathrm{a}}$ & $43.25(40.0-48.0)$ & $44.0(41.0-45.0)^{\mathrm{c}}$ \\
LDL-CHOL $(\mathrm{mg} / \mathrm{dl})$ & $99.5(91.0-109.0)^{\mathrm{a}}$ & $128.5(111.0-169.0)$ & $134.0(120.0-180.0)^{\mathrm{c}}$ \\
triglycerides $(\mathrm{mg} / \mathrm{dl})$ & $86.0(66.5-100.0)^{\mathrm{a}}$ & $144.5(116.0-199.5)^{\mathrm{b}}$ & $110(90.0-120.0)^{\mathrm{c}}$ \\
\hline
\end{tabular}

(19 patients). There were no differences in the levels of NGAL, HSP, MMP, or TIMP between these groups.

Twenty-four children with primary nocturnal enuresis and normal kidney function, served as controls.

None of the patients showed clinical evidence of infection, had diabetes, malignancies or vasculitides, smoked, took antibiotics, corticosteroids or immunosuppressive therapy. All APD children had their blood pressure values below the 90th percentile for smaller children and below 120/80 $\mathrm{mmHg}$ for adolescents, according to the criteria of the fourth report on high blood pressure in children and adolescents (National 2004), and did not require anti-hypertensives. The blood pressure in our HD patients was within normal values without medication (12 patients) or was well controlled with the use of ACE inhibitors only (three) or ACE inhibitors with calcium channel blockers (two).

Informed consent was obtained from the subjects and their parents, if necessary. The research project has been approved by the University ethics committee, in accordance with the Helsinki declaration.

Blood samples were drawn after an overnight fast from peripheral veins in APD patients and controls, in HD subjects-from the afferent line of the first-use dialyzer before starting an HD session. Samples were clotted for $30 \mathrm{~min}$, centrifuged at $4^{\circ} \mathrm{C}$ for $10 \mathrm{~min}$, and then serum was stored at $-20^{\circ} \mathrm{C}$ until assayed. Serum concentrations of NGAL, MMP-7 (matrilysin), MMP-9 (gelatinase B), and TIMP-1 were evaluated by commercially available ELISA kits (R\&D Systems, Abingdon, UK). Standards and serum samples were transferred to 96 well microplates pre-coated with recombinant antibodies to human NGAL, MMP-7, MMP-9 and TIMP-1. Each sample was tested in duplicate and the arithmetical mean was considered a final result. Measurements were performed according to the manufacturer's instructions; results were calculated by reference to standard curves.
The methods of evaluation of human $\mathrm{Hsp} 90 \alpha$, antiHsp60 and sE-selectin were described in our previous publication (Musiał et al. 2009).

In all patients the lipid profile (total cholesterol, HDLcholesterol, LDL-cholesterol, triglycerides, by BioSystems, Barcelona, Spain) and hsCRP as a marker of inflammation (nephelometry by Dade Behring, Marburg, Germany) were also evaluated.

\section{Statistical analysis}

Results are expressed as median values and interquartile ranges. Differences between all groups were evaluated by using nonparametric tests (Kruskal-Wallis, Mann-Whitney $U$ ). The relations between parameters were assessed by linear regression analysis. The linear regression equations were calculated as $y=\beta x+a(y$-dependent variable, $\beta$-regression coefficient, $x$-independent variable, $a$-constant term). We presented only those equations where both regression coefficient and constant term were statistically significant. Statistical analysis was performed using the package Statistica ver. 8.0. A $p$ value $<0.05$ was considered significant.

\section{Results}

Neutrophil gelatinase-associated lipocalin, MMPs, TIMP

NGAL, MMP-7, MMP-9, and TIMP-1 concentrations were significantly increased in all dialyzed children when compared to controls $(p<0.01)$ and the levels in the HD subgroup were higher than in the APD subjects (Fig. 1a-d). In regard to TIMP-1, this increase was most evident (2.6fold), contrarily to the 1.2-fold elevation of NGAL, MMP-7 and MMP-9 in the HD subjects when compared to the APD children. 
a

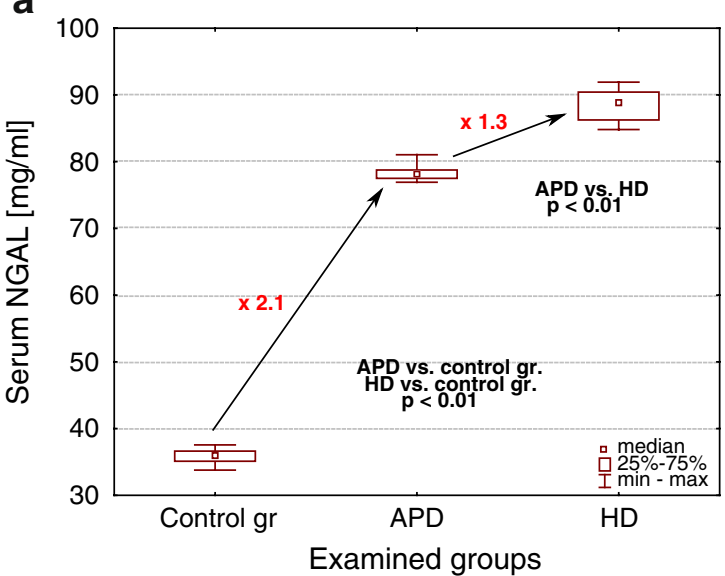

C

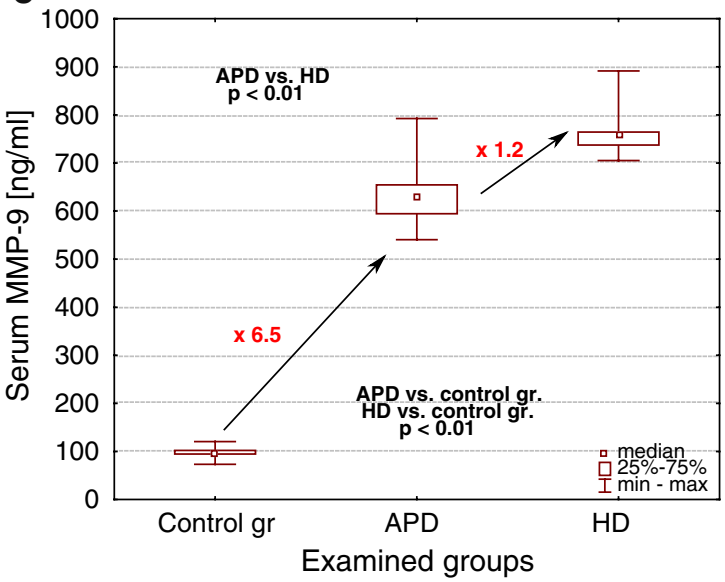

b

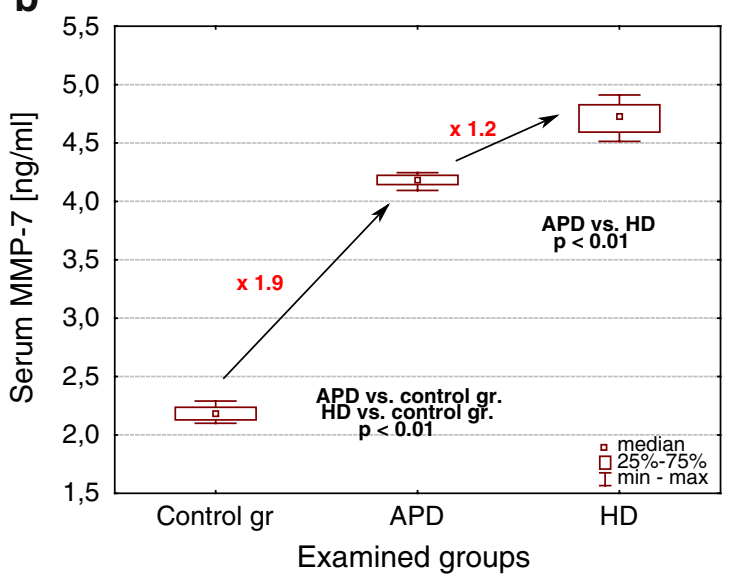

d

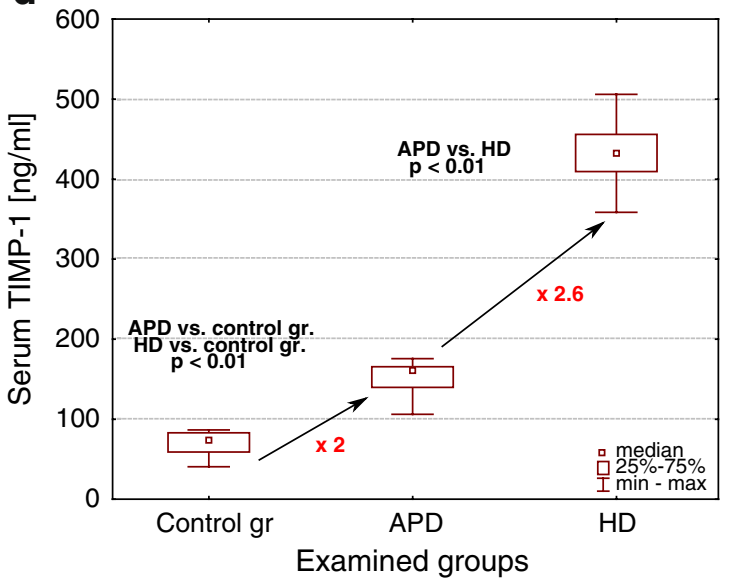

Fig. 1 The serum concentrations of examined parameters (a NGAL; b MMP-7; c MMP-9; $\mathbf{d}$ TIMP-1) in children on peritoneal dialysis (APD) on hemodialysis $(H D)$ and in the control group

Matrix metalloproteinase-7/TIMP-1, MMP-9/TIMP-1, and MMP-9/NGAL ratios

The values of MMP-7/TIMP-1 ratio were decreased in the patients on dialysis, being the lowest in the hemodialyzed subjects (Table 2). The MMP-9/TIMP-1 ratio was increased both in the APD and in the HD patients vs. controls, and the values in children on peritoneal dialysis were 2.2 times higher than those in the HD subjects (Table 2). The MMP-9/NGAL ratio was the only one that remained increased in children on dialysis in comparison to the control group and was higher in the HD patients than in the APD children (Table 2).

Table 2 The median values and interquartile ranges of the ratios of examined parameters in children on peritoneal dialysis (APD), on hemodialysis (HD) and in the control group

\begin{tabular}{llll}
\hline Parameter median values (interquartile ranges) & Control group $N=24$ & APD $N=22$ & HD $N=17$ \\
\hline MMP-9/TIMP-1 ratio & $1.43^{\mathrm{a}}(1.19-1.65)$ & $4.0^{\mathrm{b}}(3.81-4.58)$ & $1.76^{\mathrm{c}}(1.68-1.81)$ \\
MMP-7/TIMP-1 ratio & $0.030^{\mathrm{a}}(0.026-0.037)$ & $0.026^{\mathrm{b}}(0.025-0.030)$ & $0.011^{\mathrm{c}}(0.010-0.012)$ \\
MMP-9/NGAL ratio & $2.71^{\mathrm{a}}(2.61-2.96)$ & $8.08^{\mathrm{b}}(7.66-8.36)$ & $8.46^{\mathrm{c}}(8.23-8.94)$ \\
\hline
\end{tabular}

Mann-Whiney $U$ test:

${ }^{\mathrm{a}} p<0.01$ APD vs. controls

${ }^{\mathrm{b}} p<0.01$ APD vs. HD

${ }^{\mathrm{c}} p<0.01 \mathrm{HD}$ vs. controls 
Hsp90 $\alpha$, anti-Hsp60, and sE-selectin

Hsp90 $\alpha$, anti-Hsp60, and sE-selectin serum concentrations in the dialyzed population were significantly higher than in the control group (Table 1). Anti-Hsp60 and sE-selectin levels were also increased in the patients on hemodialysis when compared with those on peritoneal dialysis.

hsCRP, the lipid profile

High sensitivity CRP levels did not show significant differences between dialyzed children and controls (Table 1). Total cholesterol was significantly elevated only in the APD children (Table 1). HDL-cholesterol concentrations were decreased, whereas those of LDL-cholesterol and triglycerides were increased in patients on dialysis versus controls. However, only in the case of triglycerides there was a significant difference between the HD and APD subjects, the latter being significantly higher (Table 1)

\section{Linear regression analysis}

In all dialyzed patients, NGAL, MMPs, and TIMP-1 correlated with each other. We have also found correlations between the above mentioned parameters and other examined markers.

In detail, Hsp90 $\alpha$, anti-Hsp60 and triglycerides predicted the values of MMP-7, MMP-9, TIMP-1, MMP-7/TIMP-1, and MMP-9/TIMP-1 ratios (Tables 3 and 4).

sE-selectin, Hsp90 $\alpha$, and anti-Hsp60 were the predictors of NGAL, whereas NGAL itself predicted MMP-7, MMP9, and TIMP-1 values (Table 3), as well as MMP-7/TIMP-1 and MMP-9/TIMP-1 ratios (Table 4).
In regard to MMP-9/NGAL ratio, such a prediction concerned only anti-Hsp60 (Table 4).

No associations between MMP/TIMP/NGAL and hsCRP were observed.

\section{Discussion}

Our study describes for the first time the disturbed NGAL and MMP/TIMP systems in children with end-stage renal failure on dialysis.

The NGAL concentrations in the dialyzed patients were elevated when compared to controls and higher in the subjects on HD than in children on APD. Although the increased levels of NGAL in adults on chronic hemodialysis, as well as their correlation with inflammation markers, have been confirmed recently (Małyszko et al. 2009), there are no studies assessing lipocalin 2 in the light of different dialysis modalities. Therefore, the interpretation of elevated serum NGAL concentrations seems difficult.

Indeed, increased lipocalin 2, apart from being a marker of renal insufficiency, can also be the response to oxidative or thermal stresses (Roudkenar et al. 2007; Roudkenar et al. 2009). It was shown that NGAL expression increased in a mouse kidney exposed to cold or heat stress (Roudkenar et al. 2009). Moreover, the in vitro investigation on human embryonic kidney cells (HEK293T) revealed the protective and anti-apoptotic role of NGAL against thermal stress. In detail, the addition of recombinant NGAL to HEK293T, prior to cold stress or after heat exposure, protected those cells against the stress-induced apoptosis (Roudkenar et al. 2009). Thus, it was proven that NGAL behaved like a classical stress protein. From this point of view, the difference
Table 3 The statistically significant correlations between the examined parameters assessed by linear regression analysis in all children and young adults on dialysis (APD+HD)

\begin{tabular}{llcrrr}
\hline $\begin{array}{l}\text { Dependent } \\
\text { variable }\end{array}$ & $\begin{array}{l}\text { Independent } \\
\text { variable }\end{array}$ & $\begin{array}{l}\text { Regression } \\
\text { coefficient } \beta\end{array}$ & $\begin{array}{l}\text { Constant } \\
\text { term }\end{array}$ & $\begin{array}{l}\text { 95\% confidence } \\
\text { interval (CI) }\end{array}$ & $p$ \\
\hline MMP-7 (ng/ml) & Hsp90 $\alpha(\mathrm{ng} / \mathrm{ml})$ & 0.007 & 3.99 & $4.19-4.29$ & 0.00 \\
& Anti-Hsp60 (ng/ml) & 0.004 & 4.09 & $4.35-4.52$ & 0.00 \\
& Triglycerides (mg/dl) & -0.002 & 4.71 & $4.36-4.55$ & 0.02 \\
& NGAL (ng/ml) & 0.03 & 1.74 & $4.25-4.39$ & 0.00 \\
MMP-9 (ng/ml) & Hsp90 $\alpha(\mathrm{ng} / \mathrm{ml})$ & 1.49 & 595.19 & $624.79-670.16$ & 0.00 \\
& Anti-Hsp60 (ng/ml) & 1.17 & 602.04 & $667.49-717.35$ & 0.00 \\
& Triglycerides (mg/dl) & -0.55 & 763.04 & $671.33-722.71$ & 0.03 \\
& NGAL (ng/ml) & 6.72 & 135.70 & $642.85-690.85$ & 0.00 \\
TIMP-1 (ng/ml) & Hsp90 $\alpha(\mathrm{ng} / \mathrm{ml})$ & 3.38 & 61.14 & $160.68-198.04$ & 0.00 \\
& Anti-Hsp60 (ng/ml) & 2.30 & 99.28 & $240.45-315.32$ & 0.00 \\
& Triglycerides (mg/d) & -1.19 & 440.15 & $250.72-342.19$ & 0.01 \\
& NGAL $(\mathrm{ng} / \mathrm{ml})$ & 15.54 & -999.39 & $189.88-266.26$ & 0.00 \\
NGAL (ng/ml) & Hsp90 $\alpha(\mathrm{ng} / \mathrm{ml})$ & 0.13 & 74.63 & $78.33-79.80$ & 0.00 \\
& Anti-Hsp60 (ng/ml) & 0.06 & 77.25 & $79.29-83.84$ & 0.03 \\
& sE-selectin (pg/ml) & 0.21 & 61.47 & $79.55-82.98$ & 0.01 \\
\hline
\end{tabular}


Table 4 The statistically significant correlations between the ratios of examined parameters assessed by linear regression analysis in all children and young adults on dialysis $(\mathrm{APD}+\mathrm{HD})$

\begin{tabular}{lllccc}
\hline $\begin{array}{l}\text { Dependent } \\
\text { variable }\end{array}$ & $\begin{array}{l}\text { Independent } \\
\text { variable }\end{array}$ & $\begin{array}{l}\text { Regression } \\
\text { coefficient } \beta\end{array}$ & $\begin{array}{l}\text { Constant } \\
\text { term }\end{array}$ & $\begin{array}{l}\text { 95\% confidence } \\
\text { interval }(\mathrm{CI})\end{array}$ & $p$ \\
\hline MMP-7/TIMP-1 ratio & Hsp90 $\alpha(\mathrm{ng} / \mathrm{ml})$ & -0.0002 & 0.03 & $0.024-0.028$ & 0.00 \\
& Anti-Hsp60 $(\mathrm{ng} / \mathrm{ml})$ & -0.0001 & 0.03 & $0.018-0.023$ & 0.00 \\
& Triglycerides $(\mathrm{mg} / \mathrm{dl})$ & 0.0001 & 0.01 & $0.016-0.022$ & 0.02 \\
& NGAL $(\mathrm{ng} / \mathrm{ml})$ & -0.0009 & 0.09 & $0.021-0.027$ & 0.00 \\
MMP-9/TIMP-1 ratio & Hsp90 $\alpha(\mathrm{ng} / \mathrm{ml})$ & -0.03 & 5.06 & $3.67-4.33$ & 0.00 \\
& Anti-Hsp60 $(\mathrm{ng} / \mathrm{ml})$ & -0.02 & 4.74 & $2.74-3.51$ & 0.00 \\
& Triglycerides $(\mathrm{mg} / \mathrm{dl})$ & 0.01 & 1.82 & $2.54-3.41$ & 0.02 \\
& NGAL $(\mathrm{ng} / \mathrm{ml})$ & -0.13 & 14.14 & $3.17-3.92$ & 0.00 \\
MMP-9/NGAL ratio & Anti-Hsp60 $(\mathrm{ng} / \mathrm{ml})$ & 0.009 & 7.79 & $8.15-8.88$ & 0.03 \\
\hline
\end{tabular}

between patients on peritoneal dialysis and hemodialysis would confirm that the latter is more stress-inducing.

The discrepancy in NGAL elevation between two different modalities could also result from the neutrophil sequestration, taking place during every hemodialysis session (Andreoli et al. 2007). Another interesting finding was the existence of correlations between NGAL and heat shock proteins. The possible explanation for such connection might be the impact of NF- $\mathrm{KB}$, which is upregulated by HSPs and then may trigger NGAL activity (Bu et al. 2006; Cowland et al. 2006). Additionally, the prediction of lipocalin 2 values by sE-selectin may point to relations between NGAL and endothelial damage. The intriguing ability of NGAL, similar to that of Hsp90 $\alpha$ and anti-Hsp60, to predict MMP-7, MMP-9, and TIMP-1 concentrations in the whole population on dialysis, suggests that lipocalin 2 may share some common features with HSPs. Whether this could be another proof of its activity as a stress marker, seems so far an open question that needs further in-depth investigation before it can be definitely answered.

On the other hand, NGAL, by the blockage of MMP-9, prevents it from degradation. Therefore, the MMP-9 elevation, observed in our patients, may, at least partially, depend on the NGAL increase. Whatever the underlying cause is, elevated serum MMP-9 concentrations, observed in all dialyzed children, suggest the proteolytic overactivity in that group of patients. The potential dialysis-related trigger factors responsible for such conditions are multiple, since close relations of MMP/TIMP system with oxidative stress and inflammation in patients on hemodialysis have been described recently (Pawlak et al. 2005). The correlation with fibrotic processes has also been suggested (Rysz et al. 2010), although the authors observed decreased MMP-9 levels in adults on hemodialysis. The cause for such discrepancies may lie in the number of examined patients, not exceeding 20 subjects in all cited papers. However, in the case of children such situation is conditioned by the small size of overall pediatric population on dialysis. Another interesting finding was the difference in MMP-9 concentrations between HD and APD subjects. There is only one study (Ebihara et al. 1998) that describes the increased levels of MMP-9 mRNA in peripheral blood monocytes of dialyzed patients, being higher in HD patients than in CAPD subjects and, thus, confirming our results. The differences between dialysis modalities may also be explained, at least in part, by the results of the in vitro and animal studies. Misra et al. have described the upregulation of MMP-9, induced by the wall shear stress, in the hemodialysis grafts of rats (Misra et al. 2008). Reno et al. have shown the increased granulocyte adhesion and MMP9 release due to the contact with artificial surface, thus suggesting the possible impact of the dialyzer in the HD patients on the elevated gelatinase $\mathrm{B}$ concentrations, when compared with the APD subjects (Reno et al. 2004). Last, but not least, the already mentioned impact of NGAL may be of importance. The influence of lipocalin 2 may partially explain both the difference between dialysis modalities and the fact that the MMP-9 concentrations in children on APD exceeded those in controls nearly 7-fold, whereas in the case of MMP-7, TIMP-1 or NGAL this rise was only 2fold. We have also observed that Hsp90 $\alpha$ and anti-Hsp60 could predict the values of MMP-9, indicating its potential role as a stress marker in the dialyzed pediatric population. This finding is a novelty, not reported so far except for our similar observations concerning children with chronic kidney disease in a pre-dialysis state (Musiał et al. CSAC 2010). The reason for such a connection may be the functional chain, starting with HSPs that trigger nuclear factor (NF)-KB activity, which in turn upregulates MMPs and TIMP1 (Schett et al. 1995; Madrigal-Matute et al. 2010). The same correlation in the case of triglycerides slightly suggests the possible connection of MMP-9 with atherogenesis.

The data on MMP-7 are based mainly on animal investigation, showing its pro-atherogenic activity in the tissue remodeling in heart failure and the reduction of smooth muscle content in atherosclerotic plaque (Boixel et al. 2003; Johnson et al. 2005). The study in patients with coronary artery disease (CAD) has revealed the increased MMP-7 
concentrations, unrelated to the inflammation markers, in both stable and unstable CAD (Nilsson et al. 2006). Nothing is known about matrilysin in patients with chronic kidney disease. Our study has shown its increased concentrations in children and young adults on dialysis, the values in the HD subjects being higher than those in the APD children, similarly to MMP-9. Therefore, together with the observed correlations between MMP-7, heat shock proteins and triglycerides, we can cautiously speculate once again on the possible role of matrilysin as a new marker of stress response and atherogenesis.

TIMP-1 concentrations rose in both APD and HD subjects, although the difference in favor of HD (2.6-fold higher than APD) was more evident than in the case of other examined molecules (MMP-7 and MMP-9 levels were 1.2-1.3-fold higher in HD than in APD). Our previous results concerning CKD children treated conservatively have shown that TIMP-1 elevation appears in the late stages of CKD, being preceded by the MMP-9 increase (Musiał et al. CSAC 2010). Therefore, we can suspect that growing TIMP1 concentrations compensate the changes in MMP-9 levels. Possibly, the increase triggered during the hemodialysis session, caused by additional release of TIMP-1 from platelets, could be the explanation for the fact that the difference between APD and HD is more evident than in the case of MMPs or NGAL (Villeneuve et al. 2009). Similarly to MMPs, prediction of TIMP-1 by HSPs and triglycerides implies the possibility of its application as stress and atherogenesis- related marker.

Taken together, the fact that NGAL, MMP-9, and MMP7 values were all 1.2-fold higher in patients on hemodialysis than in the subjects dialyzed peritoneally, may suggest the common pathway of such elevation, possibly reliant on the blood-dialyzer contact.

Finally, we decided to analyze the net effect of MMP/TIMP/ NGAL activity by evaluating the balance between MMPs, their inhibitor and NGAL. Therefore, we assessed the values of ratios between selected parameters. The median values of MMP-7/TIMP-1 and MMP-9/TIMP-1 ratios were higher in APD than in HD patients. In both cases TIMP-1 elevation in HD patients was more pronounced than that of MMP-7 and MMP-9, resulting in the decrease of ratio value vs. APD children. This might be due to the previously mentioned release of TIMP-1 from platelets during hemodialysis. It may also mean that high activity of metalloproteinases, pronounced on hemodialysis, requires more intense suppression. The median values of MMP-9/NGAL ratio were higher in the dialyzed population than in controls, due to MMP-9 elevation (6.5-fold) dominant over NGAL increase (2.1-fold). As expected, Hsp90 $\alpha$, anti-Hsp60, and triglycerides were predictors of MMP-7/TIMP-1 and MMP-9/TIMP-1 ratio values.

On the whole, the values of all examined parameters were elevated in patients on dialysis compared to controls.
Moreover, the comparison of MMP/TIMP values between previously evaluated CKD children (Musiał et al. CSAC 2010) and presently examined population revealed that the concentrations in dialyzed subjects were significantly higher. This observation confirms the impact of dialysis per se on the examined parameters. Besides, the values of assessed markers remained higher on hemodialysis than in children on peritoneal dialysis. Therefore, we may hypothesize that although proteolytic overactivity is seen in every subject on dialysis, it prevails in HD. Since peritoneal dialysis is the first choice therapy in CKD children, our results are of clinical importance, speaking in favor of PD as a method triggering proteolytic activity and stress reactions to a lesser extent.

Additionally, the correlations we observed between examined parameters, heat shock proteins and triglycerides, suggest the probable role for components of NGAL/MMP/ TIMP system as possible markers of stress response and atherosclerosis development.

Finally, we are aware of the limitations of our study. Due to the fact that there are no comparative data on relations between NGAL, MMPs, TIMPs, and HSPs, our hypotheses and conclusions had to be cautious. The multiplicity of correlations between examined parameters strongly suggests the existence of a functional network, probably engaging other factors that we have not assessed. Moreover, the size of evaluated groups, conditioned by the overall small population of children on dialysis, restricts the power of conclusions that can be drawn. Therefore, the meaning of the interplay between matrix metalloproteinases and heat shock proteins, as well as the role of NGAL as a novel stress marker, needs further investigation.

\section{Conclusions}

The increased concentrations of examined parameters suggest the disturbances in NGAL/MMP/TIMP system in pediatric population with end-stage renal disease on chronic dialysis. Those abnormalities are more pronounced in patients on hemodialysis than in those dialyzed peritoneally. Our investigation has revealed that NGAL, correlating with heat shock proteins and sharing common features with them, may serve as a novel stress marker in children and young adults chronically dialyzed. Correlations between MMP/TIMP, HSPs, and triglycerides suggest that MMP-7, MMP-9, and TIMP-1 may also be regarded as indicators of stress response and atherosclerosis development in pediatric population on chronic dialysis.

Open Access This article is distributed under the terms of the Creative Commons Attribution Noncommercial License which permits any noncommercial use, distribution, and reproduction in any medium, provided the original author(s) and source are credited. 


\section{References}

Andreoli MC, Dalboni MA, Watanabe R et al (2007) Impact of dialyzer membrane on apoptosis and function of polymorphonuclear cells and cytokine synthesis by peripheral blood mononuclear cells in hemodialysis patients. Artif Organs 31:887-910

Blasi C (2008) The autoimmune origin of atherosclerosis. Atherosclerosis 201:17-32

Boixel C, Fontaine V, Rücker-Martin C et al (2003) Fibrosis of the left atria during progression of heart failure is associated with increased matrix metalloproteinases in the rat. J Am Coll Cardiol 42:336-344

Bolignano D, Lacquaniti A, Coppolino G, Campo S, Arena A, Buemi M (2008) Neutrophil gelatinase-associated lipocalin reflects the severity of renal impairment in subjects affected by chronic kidney disease. Kidney Blood Press Res 31:255-258

Bu DX, Hemdahl AL, Gabrielsen A, Fuxe J, Zhu Ch, Eriksson P, Yan ZQ (2006) Induction of neutrophil gelatinase-associated lipocalin in vascular injury via activation of nuclear factor- $\mathrm{kB}$. Am J Pathol 169:2245-2253

Cappello F, de Macario EC, Di Felice V, Zummo G, Macario AJL (2009) Chlamydia trachomatis infection and anti-Hsp60 immunity: the two sides of the coin. PLoS Pathog 8:e1000552

Cowland JB, Muta T, Borregaard N (2006) IL-1beta-specific upregulation of neutrophil gelatinase-associated lipocalin is controlled by IкB-zeta. J Immunol 176:5559-5566

Diamanti-Kandarakis E, Livadas S, Kandarakis SA, Margeli A (2008) Serum concentrations of atherogenic proteins neutrophil gelatinase-associated lipocalin and its complex with matrix metalloproteinase-9 are significantly lower in women with polycystic ovary syndrome: hint of a protective mechanism? Eur J Endocrinol 158:525-531

de Nooijer R, Verkleij CJN, von der Thusen HJ et al (2006) Lesional overexpression of matrix metalloproteinase- 9 promotes intraplaque hemorrhage in advanced lesions but not earlier stages of atherogenesis. Arterioscler Thromb Vasc Biol 26:340-346

Dulin E, Garcia-Barreno P, Guisasola MC (2010) Extracellular heat shock protein 70 (HSPA1A) and classical vascular risk factors in a general population. Cell Stress Chaperones (in press). doi:10.1007/s12192-010-0201-2

Ebihara I, Nakamura T, Tomino Y, Shimada N, Koide H (1998) Metalloproteinase-9 mRNA expression in monocytes from patients with chronic renal failure. Am J Nephrol 18:305-310

Fukudome K, Fujimoto S, Sato Y, Hisanaga S, Eto T (2001) Peritonitis increases MMP-9 activity in peritoneal effluent from CAPD patients. Nephron 87:35-41

Hansson J, Lind L, Hulthe J, Sundstrom J (2009) Relations of serum MMP-9 and TIMP-1 levels to left ventricular measures and cardiovascular risk factors: a population-based study. Eur J Cardiovasc Prev Rehab 16:297-303

Hemdahl AL, Gabrielsen A, Zhu C, Eriksson P, Hedin U, Kastrup J, Thoren P, Hansson GK (2006) Expression of neutrophil gelatinase-associated lipocalin in atherosclerosis and myocardial infarction. Arterioscler Thromb Vasc Biol 26:136-142

Jefferis BJ, Whincup P, Welsh P et al (2010) Prospective study of matrix metalloproteinase- 9 and risk of myocardial infarction and stroke in older men and women. Atherosclerosis 208:557-563

Johnson JL, George SJ, Newby AC, Jackson CL (2005) Divergent effects of matrix metalloproteinases $3,7,9$, and 12 on atherosclerotic plaque stability in mouse brachiocephalic arteries. Proc Natl Acad Sci USA 102:15575-15580

Kuzuya M, Nakamura K, Sasaki T et al (2006) Effect of MMP-2 deficiency on atherosclerotic lesion formation in apoE-deficient mice. Arterioscler Thromb Vasc Biol 26:1120-1125

Leng X, Zhan R, Wang Y et al (2010) Anti-heat shock protein 70 autoantibody epitope changes and BD091 promotes atheroscle- rosis in rats. Cell Stress Chaperones (in press). doi:10.1007/ s12192-010-0203-0

Leung JC, Lam MF, Tang SC, Chan LY, Tam KY, Yip TP, Lai KN (2009) Roles of neutrophil gelatinase-associated lipocalin in continuous ambulatory peritoneal dialysis-related peritonitis. J Clin Immunol 29:365-378

Luttun A, Lutgens E, Manderveld A et al (2004) Loss of matrix metalloproteinase- 9 or matrix metalloproteinase- 12 protects apolipoprotein E-deficient mice against atherosclerotic media destruction but differentially affects plaque growth. Circulation 109:1408-1414

Madrigal-Matute J, Lopez-Franco O, Blanco-Colio LM et al (2010) Heat shock protein 90 inhibitors attenuate inflammatory responses in atherosclerosis. Cardiovasc Res 86(2):330-337. doi: $10.1093 / \mathrm{cvr} / \mathrm{cvq} 046$

Małyszko J, Małyszko JS, Koc-Żórawska E, Koźmiński P, Myśliwiec M (2009) Neutrophil gelatinase-associated lipocalin in dialyzed patients is related to residual renal function, type of renal replacement therapy and inflammation. Kidney Blood Press Res 32:464-469

Misra S, Fu AA, Puggioni A et al (2008) Increased shear stress with upregulation of VEGF-A and its receptors and MMP-2, MMP-9, and TIMP-1 in venous stenosis of hemodialysis grafts. Am J Physiol Heart Circ Physiol 294:H2219-H2230

Mitsnefes MM, Kathman ThS, Mishra J et al (2007) Serum neutrophil gelatinase-associated lipocalin as a marker of renal function in children with chronic kidney disease. Pediatr Nephrol 22:101108

Musiał K, Szczepańska M, Szprynger K, Zwolińska D (2009) The impact of dialysis modality on serum heat shock proteins in children and young adults with chronic kidney disease. Kidney Blood Press Res 32:366-372

Musiał K, Zwolińska D (2010) Matrix metalloproteinases (MMP-2,9) and their tissue inhibitors (TIMP-1,2) as novel markers of stress response and atherogenesis in children with chronic kidney disease (CKD) on conservative treatment. Cell Stress Chaperones (in press). doi:10.1007/s12192-010-0214-x

Naganuma T, Sugimura K, Uchida J, Tashiro K, Yoshimura R, Takemoto Y, Nakatani T (2008) Increased levels of serum matrix metalloproteinase-3 in haemodialysis patients with dialysisrelated amyloidosis. Nephrology 13:104-108

National High Blood Pressure Education Program Working Group on High Blood Pressure in Children and Adolescents (2004) The fourth report on the diagnosis, evaluation, and treatment of high blood pressure in children and adolescents. Pediatrics 114:555576

Newby CN, George SJ, Ismail Y, Johnson JL, Sala-Newby GB, Thomas AC (2009) Vulnerable atherosclerotic plaque metalloproteinases and foam cell phenotypes. Thromb Haemost 101:1006-1011

Nilsson L, Jonasson L, Nijm J, Hamsten A, Eriksson P (2006) Increased plasma concentration of matrix metalloproteinase-7 in patients with coronary artery disease. Clin Chem 52:1522-1527

Nishida M, Kawakatsu H, Okumura Y, Hamaoka K (2010) Serum and urinary NGAL levels in children with chronic renal diseases. Pediatr Int (in press)

Pawlak K, Pawlak D, Myśliwiec M (2005) Circulating beta-chemokines and matrix metalloproteinase-9/tissue inhibitor of metalloproteinase-1 system in hemodialyzed patients - role of oxidative stress. Cytokine 31:18-24

Perschinka H, Mayr M, Millonig G, Mayerl Ch, Van der Zee R, Morrison SG, Morrison RP, Xu Q, Wick G (2003) Cross-reactive B-cell epitopes of microbial and human heat shock protein $60 / 65$ in atherosclerosis. Arterioscler Thromb Vasc Biol 23:1060-1065

Pockley AG, Bulmer J, Hanks BM, Wright BH (1999) Identification of human heat shock protein 60 (Hsp60) and anti-Hsp60 
antibodies in the peripheral circulation of normal individuals. Cell Stress Chaperones 4:29-35

Preston GA, Barrett CV, Alcorta DA et al (2002) Serum matrix metalloproteinases MMP-2 and MMP-3 levels in dialysis patients vary independently of CRP and IL-6 levels. Nephron 92:817-823

Reno F, Lombardi F, Cannas M (2004) Polystyrene surface coated with vitamin $\mathrm{E}$ modulates human granulocyte adhesion and MMP-9 release. Biomol Eng 21:73-80

Rigano R, Profumo E, Buttari B et al (2007) Heat shock proteins and autoimmunity in patients with carotid atherosclerosis. Ann NY Acad Sci 1107:1-10

Roudkenar MH, Kuwahara Y, Baba T, Roushandeh AM, Ebishima S, Abe S, Ohkubo Y, Fukumoto M (2007) Oxidative stress induced lipocalin 2 gene expression: addressing its expression under the harmful conditions. J Radiat Res 48:39-44

Roudkenar MH, Halabian R, Roushandeh AM et al (2009) Lipocalin 2 regulation by thermal stresses: protective role of Lcn2/NGAL against cold and heat stresses. Exp Cell Res 315:3140-3151
Rysz J, Banach M, Stolarek RA et al (2010) Serum metalloproteinases MMP-2, MMP-9 and metalloproteinase tissue inhibitors TIMP-1 and TIMP-2 in patients on hemodialysis. Int Urol Nephrol (in press). doi:10.2007/s11255-009-9683-1

Schett G, Xu Q, Amberger A, Van der Zee R, Recheis H, Willeit J, Wick G (1995) Autoantibodies against heat shock protein 60 mediate endothelial cytotoxicity. J Clin Invest 96:2569-2577

Villeneuve J, Block A, Le Bousse-Kerdiles MC et al (2009) Tissue inhibitors of matrix metalloproteinases in platelets and megakaryocytes: a novel organization for these secreted proteins. Exp Hematol 37:849-856

Wick G, Knoflach M, Xu Q (2004) Autoimmune and inflammatory mechanisms in atherosclerosis. Annu Rev Immunol 22:361-403

Wu T, Tanguay RM (2006) Antibodies against heat shock proteins in environmental stresses and diseases: friend or foe? Cell Stress Chaperones 11:1-12

Zhang X, Xu Z, Zhou L et al (2010) Plasma levels of Hsp70 and antiHsp70 antibody predict risk of acute coronary syndrome. Cell Stress Chaperones 15(5):675-686. doi:10.1007/s12192-010-0180-3 